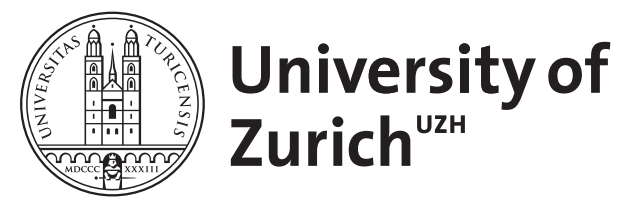

\title{
Oscillations of the ${ }^{7} \mathrm{Be}$ solar neutrinos inside the Earth
}

\author{
Ioannisian, A N ; Smirnov, A Y ; Wyler, D
}

\begin{abstract}
We explore in detail oscillations of solar ${ }^{7} B e$ neutrinos passing through the Earth. The depth of oscillations is at the (0.1-0.2)\% level, and the length is approximately $30 \mathrm{~km}$. The change of the oscillatory modulations with energy is comparable to the width of the ${ }^{7} \mathrm{Be}$ line, which is determined by the temperature in the center of the Sun. This means that, depending on the length of the trajectory (nadir angle), one obtains different degrees of cancellations when averaging the oscillations over the ${ }^{7} B e$ neutrino energy. Thus, by exploring these oscillations in detail, it is possible to determine the width of the ${ }^{7} \mathrm{Be}$ line and therefore the temperature of the Sun and to fix $\Delta m_{21}^{2}$ precisely. Furthermore, it allows us to perform tomography of the Earth, in particular to measure the deviation from a spherical mass distribution and detect small inhomogeneities. Studies of the Be neutrinos open up a possibility to test quantum mechanics of neutrino oscillations and search for sterile neutrinos. We also estimate the accuracy of these measurements with future scintillator (or scintillator uploaded) detectors of $\sim 100 \mathrm{kton}$ mass.
\end{abstract}

DOI: https://doi.org/10.1103/PhysRevD.92.013014

Posted at the Zurich Open Repository and Archive, University of Zurich ZORA URL: https://doi.org/10.5167/uzh-121349

Journal Article

Accepted Version

Originally published at:

Ioannisian, A N; Smirnov, A Y; Wyler, D (2015). Oscillations of the ${ }^{7} B e$ solar neutrinos inside the Earth. Physical Review D (Particles, Fields, Gravitation and Cosmology), 92:013014.

DOI: https://doi.org/10.1103/PhysRevD.92.013014 


\title{
Oscillations of the ${ }^{7}$ Be solar neutrinos inside the Earth
}

\author{
A. N. Ioannisian ${ }^{1,2}$, A. Yu. Smirnov ${ }^{3,4}$, D. Wyler ${ }^{5}$ \\ 1 Yerevan Physics Institute, Alikhanian Br. 2, 375036 Yerevan, Armenia \\ ${ }^{2}$ Institute for Theoretical Physics and Modeling, 375036 Yerevan, Armenia \\ 3 Max-Planck Institute for Nuclear Physics, Saupfercheckweg 1, D-69117 Heidelberg, Germany \\ ${ }^{4}$ ICTP, Strada Costiera 11, 34014 Trieste, Italy \\ 5 Institut für Theoretische Physik, Universität Zürich, \\ Winterthurerstrasse 190, CH-8057 Zürich, Switzerland
}

\begin{abstract}
We explore in detail oscillations of the solar ${ }^{7}$ Be neutrinos in the matter of the Earth. The depth of oscillations is about $(0.1-0.2) \%$ and the length $\approx 30 \mathrm{~km}$. The period of the oscillatory modulations in the energy scale is comparable with the width of the line determined by the temperature in the center of the Sun. The latter means that depending on the length of trajectory (nadir angle) one obtains different degree of averaging of oscillations. Exploring these oscillations it is possible to measure the width of the ${ }^{7} \mathrm{Be}$ line and therefore the temperature of the Sun, determine precisely $\Delta m_{21}^{2}$, perform tomography of the Earth, in particular, measure the deviation of its form from sphere, and detect small structures. Studies of the Be neutrinos open up a possibility to test quantum mechanics of neutrino oscillations and search for the sterile neutrinos. Accuracy of these measurements with future scintillator (or scintillator uploaded) detectors of the $\sim 100$ kton mass scale is estimated.
\end{abstract}

PACS numbers: 14.60.Pq, 26.65.+t, 95.85.Ry, 95.60.Jw,

\section{INTRODUCTION}

Exploration of the solar neutrinos moves to the phase of precision measurements. In future, developments of the detection techniques and construction of the large mass detectors can open up various new possibilities and one of them is study of the solar ${ }^{7} \mathrm{Be}$ neutrino oscillations in the Earth.

The solar ${ }^{7} \mathrm{Be}$ neutrinos have two salient properties: low energies and narrow width. Due to environment effects in the Sun [1] the energy profile of the ${ }^{7}$ Be neutrinos, $g(E)$, is broadened and has an asymmetric form. The maximum value of $g(E), E \sim 862.27 \mathrm{keV}$, is shifted to higher energy with respect to the laboratory value $E_{l a b} \sim$ $861.64 \mathrm{keV}$ [2]. The left (low energy) wing of the profile is determined by the Doppler shift caused by thermal velocities of ${ }^{7} \mathrm{Be}$ nuclei. The right (high energy) wing is mainly due to collisions determined by temperature at the center of the Sun (averaged over ${ }^{7} \mathrm{Be}$ neutrinos production region). The width of the line at the half of height equals

$$
\Gamma_{\mathrm{Be}} \simeq 1.6 \mathrm{keV},
$$

so that the relative size of the width is

$$
\frac{\Gamma_{\mathrm{Be}}}{E}=1.86 \cdot 10^{-3} \text {. }
$$

The width and the shift of maximum of $g(E)$ are proportional to the central temperature of the Sun $T_{c}$. In [1] it was proposed to determine $T_{c}$ by measuring the shift of the peak. It was also mentioned that finite width of the ${ }^{7} \mathrm{Be}$ spectrum affects the depth of vacuum oscillations.

According to the standard solar model the flux of ${ }^{7} \mathrm{Be}$ neutrinos is known with $1.4 \%$ accuracy. For the LMA
MSW values of oscillation parameters the ${ }^{7}$ Be neutrino line is at the low energy edge of the so called transition region between the averaged vacuum oscillations and matter dominated conversion. The flux is suppressed mainly by the averaged vacuum oscillations probability with small additional suppression due to matter effect in the Sun.

The ${ }^{7}$ Be neutrino flux measured by BOREXINO [3], [4] and KamLAND [5] is in a good agreement with the SSM predicted flux with suppression according the LMA solution. In fact, these measurements (reached $5 \%$ accuracy) provided important confirmation and consistency checks of the LMA MSW solution of the solar neutrino problem. Time dependence of the flux has been explored [6]. The data are in agreement with seasonal variations due to eccentricity of the Earth orbit and no other variations have been found in agreement with LMA MSW expectations. In particular, after 2 years of exposure and 0.133 kton fiducial mass the following bound on the Day - Night asymmetry has been obtained [6]:

$$
A_{d n}=2 \frac{R_{N}-R_{D}}{R_{N}+R_{D}}=0.001 \pm 0.012 \text { (stat) } \pm 0.007 \text { (syst). }
$$

On the way from the Sun the coherence of neutrino state is lost, so that incoherent fluxes of the mass states arrive at the surface of the Earth. Inside the Earth these mass states oscillate due to matter effect. According to LMA MSW solution these oscillations proceed in the low matter density regime, and the expected effects are very small (see e.g. [7], [8] [9], [10], [11], [12], [13], [14]). The 
effects are determined by the parameter:

$\epsilon \equiv \frac{2 V_{e} E}{\Delta m_{21}^{2}} \approx 2.4 \cdot 10^{-3}\left(\frac{\rho}{2.7 \frac{\mathrm{g}}{\mathrm{cm}^{3}}}\right)\left(\frac{7.5 \cdot 10^{-5} \mathrm{eV}^{2}}{\Delta m_{21}^{2}}\right)\left(\frac{Y_{e}}{0.5}\right)$,

where $E$ is the neutrino energy, $V_{e}=\sqrt{2} G_{F} n_{e}$ is the matter potential with $G_{F}$ and $n_{e}$ being the Fermi coupling constant and the electron number density correspondingly. The parameter $\epsilon$ characterizes deviations of the mixing angle and the oscillation length in matter from their vacuum values: $\theta_{12}$ and

$$
l_{\nu} \equiv \frac{4 \pi E}{\Delta m_{21}^{2}} \approx 28.5 \mathrm{~km}\left(\frac{7.5 \times 10^{-5} \mathrm{eV}^{2}}{\Delta m_{21}^{2}}\right) .
$$

In fact, $\epsilon$ determines the depth of oscillations of neutrinos with definite mass, $\approx 0.5 \epsilon$ which is about $\sim 0.1 \%$ in the mantle and $\sim 0.2 \%$ in the core. Being of the order $10^{-3}$ the expected effects are far beyond the present BOREXINO (3) as well as expected SNO+ sensitivities.

Next generation of large (several tenth of ktons to hundred ktons) scintillator detectors like JUNO [15] or LENA [16] will have sub-percent sensitivity to the DayNight asymmetry. Higher sensitivity can be achieved with 100 kton mass scale scintillator uploaded water detectors, WBLS [17]. For 100 kton fiducial mass and 5 years exposure such a detector will collect $1.9 \cdot 10^{3}$ bigger statistics than the one used for the result (3). Correspondingly, the statistical error will be reduced down to $3 \cdot 10^{-4}$. So, if systematic errors is well controlled, the $0.1 \%$ size Earth matter effects on the ${ }^{7}$ Be neutrinos can be established at about $3 \sigma$ level.

In this connection we will explore in detail the ${ }^{7} \mathrm{Be}$ neutrino oscillations in the matter of the Earth. There are two very interesting coincidences related to the energy and width of the ${ }^{7} \mathrm{Be}$ neutrino profile which allow one to obtain in principle unique information about neutrino properties, characteristics of the ${ }^{7} \mathrm{Be}$ neutrino spectrum, properties of the Earth density profile and quantum mechanics of neutrino oscillations. We estimate possibilities of future large detectors to determine the width of the line, and consequently, the central temperature of the Sun), to measure $\Delta m_{21}^{2}$ with unprecedent accuracy, to perform tomography of the Earth and search for very light sterile neutrinos.

The paper is organized as follows. In Sec. 2 we present relevant analytic results for the probabilities of oscillations in the Sun and the Earth as well as compute the relative variations of the flux with the nadir angle. In Sec. 3 the effects of averaging of the flux over the ${ }^{7} \mathrm{Be}$ energy spectrum are explored. We compute the time (nadir angle) variation of number of events and estimate a potential of future $100 \mathrm{kton}$ scale detectors to establish the Earth matter effect, to measure $\Gamma_{\mathrm{Be}}$ and $\Delta m_{21}^{2}$ in Sec. 4. Searches for sterile neutrinos are considered in Sec. 5. We conclude in Sec. 6.

\section{OSCILLATION IN THE SUN AND THE EARTH}

The electron neutrino $\nu_{e}$ produced in the center of the Sun is adiabatically converted into the combination of the mass eigenstates $\nu_{1}, \nu_{2}, \nu_{3}$. The combination is determined by the mixing angles, $\theta_{12}^{m}=\theta_{12}^{m}\left(V_{e}^{0}\right)$, and $\theta_{13}^{m}\left(V_{e}^{0}\right)$ in the production point

$$
\nu_{e} \rightarrow c_{13} \cos \bar{\theta}_{12}^{m} \nu_{1}+c_{13} \sin \bar{\theta}_{12}^{m} \nu_{2}+s_{13} \nu_{3},
$$

where $c_{13} \equiv \cos \theta_{13}, s_{13} \equiv \sin \theta_{13}$, and we neglected the matter effect on 1-3 mixing, so that $\theta_{13}^{m}\left(V_{e}^{0}\right) \approx \theta_{13}$ [30]. We use the standard parametrization of the PMNS mixing matrix. In Eq. (6) $\bar{\theta}_{12}^{m}$ is the value of $1-2$ angle in matter averaged over the ${ }^{7} \mathrm{Be}$ neutrino production region. It is given by

$$
\tan ^{2} 2 \bar{\theta}_{12}^{m} \approx \tan ^{2} 2 \theta_{12}\left(1-\frac{2 c_{13}^{2} \bar{V}_{e}^{0} E}{\Delta m_{21}^{2} \cos 2 \theta_{12}}\right)^{-2},
$$

where $\bar{V}_{e}^{0}$ is the average matter potential in the production region.

On the way from the Sun to the Earth the wave packets of mass eigenstates $\nu_{i}$ spread and separate. At the production the lengths of the wave packets in the configuration space equal

$$
\sigma_{x} \approx \frac{2 \pi}{\Gamma_{B e}}=6 \cdot 10^{-8} \mathrm{~cm} .
$$

Due to spread their sizes become at the surface of the Earth

$$
\sigma_{x}^{\text {spread }}=\frac{m_{i}^{2} L_{\text {sun }} \Gamma_{B e}}{E^{3}}
$$

where $m_{i}$ is the absolute value of mass of $\nu_{i}$, and $L_{\text {sun }}$ is the distance from the Sun to the Earth. For hierarchical spectrum, $m_{2} \approx \sqrt{\Delta m_{21}^{2}}$, we obtain from (9) $\sigma_{x}^{\text {spread }}=$ $3.8 \cdot 10^{-6} \mathrm{~cm}$, (i.e. 2 orders of magnitude larger than the original size of the packet). However, separation of the wave packets of different mass eigenstates is larger:

$$
\Delta x=L_{\text {sun }} \Delta m_{21}^{2} / 2 E^{2}=2 \cdot 10^{-3} \mathrm{~cm} .
$$

Even in the case of degenerate spectrum, $m_{1} \approx m_{2} \approx 0.1$ $\mathrm{eV}$, we obtain $\sigma_{x}^{\text {spread }} / \Delta x \approx 0.25$. In addition there is also averaging of oscillations over the neutrino production region inside the Sun.

Due to loss of coherence, neutrinos arrive at the surface of the Earth as incoherent fluxes of $\nu_{1}, \nu_{2}$ and $\nu_{3}$ with relative admixtures given according to Eq. (6) by $c_{13}^{2} \cos ^{2} \bar{\theta}_{12}^{m}, c_{13}^{2} \sin ^{2} \bar{\theta}_{12}^{m}$ and $s_{13}^{2}$ correspondingly. In matter of the Earth each of these mass states splits into eigenstates in matter and oscillates.

The probability to find $\nu_{e}$ in the detector after crossing the Earth can be written as

$$
\begin{aligned}
P & =c_{13}^{2}\left(\cos ^{2} \bar{\theta}_{12}^{m} P_{1 e}+\sin ^{2} \bar{\theta}_{12}^{m} P_{2 e}\right)+s_{13}^{4} \\
& =c_{13}^{2}\left(\cos 2 \bar{\theta}_{12}^{m} P_{1 e}+c_{13}^{2} \sin ^{2} \bar{\theta}_{12}^{m}\right)+s_{13}^{4},
\end{aligned}
$$


where $P_{1 e}$ and $P_{2 e}$ are the probabilities of $\nu_{1} \rightarrow \nu_{e}$ and $\nu_{2} \rightarrow \nu_{e}$ transitions in the Earth correspondingly. Here we used the unitarity relation: $P_{1 e}+P_{2 e}+s_{13}^{2}=1$ or $P_{1 e}+P_{2 e}=c_{13}^{2}$.

The probability $P$ can be represented as

$$
P \equiv P_{D}+\Delta P
$$

where

$$
P_{D}=\frac{c_{13}^{4}}{2}\left(1+\cos 2 \bar{\theta}_{12}^{m} \cos 2 \theta_{12}\right)+s_{13}^{4}
$$

is the probability during the day when $P_{1 e}=P_{1 e}^{0}=$ $c_{13}^{2} \cos ^{2} \theta_{12}, P_{2 e}=P_{2 e}^{0}=c_{13}^{2} \sin ^{2} \theta_{12}$, and

$$
\Delta P=c_{13}^{2} \cos 2 \bar{\theta}_{12}^{m}\left(P_{1 e}-P_{1 e}^{0}\right)
$$

is the difference of the probabilities during the day and the night. The probability $P_{1 e}$ equals [9]

$$
P_{1 e} / c_{13}^{2}=\cos ^{2} \theta_{12}-\frac{1}{2} \sin ^{2} 2 \theta_{12} c_{13}^{2} \int_{0}^{L} d x V_{e}(x) \sin \phi_{x \rightarrow L}^{m},
$$

where

$$
\begin{gathered}
\phi_{x \rightarrow L}^{m}(E) \equiv \int_{x}^{L} d x \Delta_{m}(x) \\
\Delta_{m}(x) \equiv \frac{\Delta m_{21}^{2}}{2 E} \sqrt{\left[\cos 2 \theta_{12}-c_{13}^{2} \epsilon(x)\right]^{2}+\sin ^{2} 2 \theta_{12}}
\end{gathered}
$$

and $\epsilon$ is determined in (4). Here $x$ is the distance between an entrance point to the Earth and a given point of trajectory.

The oscillation length can be written as

$$
l_{m}=\frac{2 \pi}{\Delta_{m}(x)}=l_{\nu}\left[1+\cos 2 \theta_{12} c_{13}^{2} \epsilon+O\left(\epsilon^{2}\right)\right] .
$$

The matter correction, $\cos 2 \theta_{12} c_{13}^{2} \epsilon$, is of the order (12) $\cdot 10^{-3}$.

Notice that since in the Sun $P>1 / 2$, or $\cos 2 \bar{\theta}_{12}^{m}>0$, the oscillations in the Earth suppress the survival probability, $\Delta P<0$, in contrast to high energy Boron neutrinos for which $\cos 2 \bar{\theta}_{12}^{m}<0$ and partial regeneration of the $\nu_{e}$ flux occurs.

The expression in (14) is equivalent to the result of adiabatic perturbation theory [10]:

$$
\begin{aligned}
P_{1 e} / c_{13}^{2} & =\cos ^{2} \theta_{12}-\sin 2 \theta_{12}^{s} \sin 2\left(\theta_{12}^{s}-\theta_{12}\right) \sin ^{2} \phi_{0 \rightarrow L / 2} \\
& +\sin 2\left(2 \theta_{12}^{s}-\theta_{12}\right) \int_{0}^{L} d \theta_{m}(x) \cos \phi_{x \rightarrow L}^{m}
\end{aligned}
$$

where $\theta_{12}^{s}$ is the mixing angle in matter at the surface of the Earth. The angle $\theta_{12}^{s}$ is given by Eq. (7) with $V_{e}$ substituted by $V_{e}\left(\rho_{\text {surf }}\right)$. We use this formula for numerical computations inside different layers of the Earth.
Combining Eqs. (12), (13) and (14) we can write the relative variation of the $\nu_{e}$-flux due to the Earth matter effect as

$$
A_{e}^{0} \equiv \frac{\Delta P}{P_{D}}=-\frac{c_{13}^{2}}{2} f\left(\Delta m_{21}^{2}, \theta_{12}, \theta_{13}\right) \int_{0}^{L} d x V_{e}(x) \sin \phi_{x \rightarrow L}^{m},
$$

where

$$
f\left(\Delta m_{21}^{2}, \theta_{12}\right) \equiv \frac{2 \cos 2 \bar{\theta}_{12}^{m} \sin ^{2} 2 \theta_{12}}{1+\cos 2 \bar{\theta}_{12}^{m} \cos 2 \theta_{12}+2 \tan ^{4} \theta_{13}} .
$$

For $\theta_{12}=34^{\circ}$ and $\Delta m_{12}^{2}=7.5 \times 10^{-5} \mathrm{eV}^{2}$ we obtain $f\left(\Delta m_{21}^{2}, \theta_{12}\right) \simeq 0.43$; corrections due to the 1-3 mixing are below $0.1 \%$.

Quick estimation of the effect can be done for constant density profile. In this case we obtain $\phi_{x \rightarrow L}^{m}(E)=$ $\Delta_{m}^{c}(L-x)$, and in the lowest order in $\epsilon$ the Eq. (19) gives

$$
A_{e}^{0} \approx-c_{13}^{2} \epsilon f \sin ^{2} \frac{1}{2} \Delta_{m} L
$$

So, the depth of the oscillations equals $c_{13}^{2} \epsilon f \approx \epsilon f$.

Dependence of $\Delta P / P^{0}$ on the nadir angle $\eta$ is shown in Figs. 1, 2 (red lines). In our computations we used $P_{1 e}$ from Eq. (18), the spherically symmetric Earth and the 5 layers parametrization of the density profile [21] with sharp density jumps at $410 \mathrm{~km}, 660 \mathrm{~km}, 2830 \mathrm{~km}$ and $5150 \mathrm{~km}$ from the surface of the Earth. The length of the neutrino trajectory inside the Earth is given by $L=2 R_{E} \cos \eta$; so dependence on $\cos \eta$ is equivalent to the dependence on $L$ and therefore Figs. 1 and 2 reflect dependence of oscillations on distance. Trajectories with $\cos \eta>0.83$ cross the core of the Earth.

According to the Figs. 1, 2 (red lines) the depth of oscillations changes with the nadir angle which is related to breaking of adiabaticity. If the adiabaticity condition is fulfilled along whole the trajectory, the oscillation depth would be determined exclusively by the mixing angle at the surface of the Earth. (More precisely, by the mixing angle averaged over the distance of the order of oscillation length). This is satisfied for shallow trajectories with $\eta>1.21$. At $\eta \approx 1.21$ neutrinos cross the first density jump (410 km from the surface) and at $\eta \approx 1.11$ - the second one $(660 \mathrm{~km})$, where adiabaticity is broken, and consequently, the depth of oscillations increases.

The biggest change is at $\eta=0.58$, where neutrinos start to cross the core of the Earth. The depth of oscillations increases by factor of 2 . At $\eta=0.19$ neutrino trajectories cross also borders of the inner core. Breakdown of the adiabaticity and oscillations in different layers lead also to modulations of the oscillatory picture which is related to the interference of effects from different layers (see below).

The length of the deepest trajectory in the mantle is $L_{\max }=10570 \mathrm{~km}$, therefore the number of periods in 
the mantle range, $\eta>0.58$, equals

$$
\frac{x_{\max }}{l_{\nu}} \approx 370 .
$$

The number of periods for the core crossing trajectories is bigger: about 420 for the vertical direction.

\section{EFFECT OF THE BE NEUTRINO LINE WIDTH}

\section{Averaging over the energy}

Signal in the detector is determined by the variation, $A_{e}$, averaged over the energy spectrum of the ${ }^{7} \mathrm{Be}$ neutrinos. Energy resolution of a detector is much worse and therefore irrelevant for the oscillation picture. The ${ }^{7} \mathrm{Be}$ neutrino flux can be written as

$$
F_{B e}(E, t)=F_{B e}^{0}(t) g(E),
$$

where $F_{B e}^{0}(t)$ is the total flux and $g(E)$ is the energy profile normalized to 1: $\int d E g(E)=1$. Dependence of $F_{B e}^{0}$ on time is due to the eccentricity of the Earth orbit around the Sun, as well as due to rotation of the Earth itself (due to bigger distance from the Sun at night). The former $( \pm 3 \%)$ must be taken into account, the latter $(<$ $10^{-4 \%)}$ can be neglected.

Variation of the $\nu_{e}$-flux averaged over the ${ }^{7} \mathrm{Be}$ spectrum for the spherically symmetric Earth equals

$$
\bar{A}_{e}(t)=\int d E g(E) A_{e}(E, t)
$$

The dependence of $\bar{A}_{e}$ on $\eta$ is shown in Figs. 1, 2 (blue solid lines). In what follows we will present complete interpretation of the this dependence.

1. Suppression of the oscillation depth. Integration over the energy profile leads to partial suppression of the oscillation depth which depends on the nadir angle. At the same time the integration does not affect the average value of the relative variations. The change of the oscillation depth with the nadir angle is result of interesting and accidental coincidence: the width of the line, $\Gamma_{\mathrm{Be}}$, is of the order of the period of oscillatory curve in the energy scale, $\Delta E_{T}$ :

$$
\Delta E_{T} \sim \Gamma_{\mathrm{Be}}
$$

The period is defined by equality

$$
\frac{d \Phi_{m}}{d E} \Delta E_{T}=2 \pi
$$

where $\Phi_{m} \approx 2 \pi L / l_{m} \approx 2 \pi L / l_{\nu}$ is the oscillation phase. We find from (26)

$$
\Delta E_{T}=E \frac{l_{\nu}}{L}=E \frac{l_{\nu}}{2 R_{E} \cos \eta},
$$

which depends on $\eta$. Therefore relation between $\Delta E_{T}$ and $\Gamma_{\mathrm{Be}}$, and consequently, change of the degree of averaging and the depth of oscillations depend on $\eta$. For shallow trajectories (large $\eta$ ) $\Delta E_{T} \gg \Gamma_{\mathrm{Be}}$ the averaging effect is negligible. With decrease of $\eta$ the period $\Delta E_{T}$ decreases and averaging becomes stronger (see Fig. 1). For the deepest trajectories in the mantle the suppression is characterized by factor $2-3$.

In configuration space, partial averaging corresponds to partial lost of coherence during propagation inside the Earth. Indeed, the relative shift of the wave packets of the eigenstates equals

$$
\Delta x=L \frac{\Delta m^{2}}{2 E^{2}}=5 \cdot 10^{-8} \mathrm{~cm}\left(\frac{L}{10^{4} \mathrm{~km}}\right)=0.8 \sigma_{x}\left(\frac{L}{10^{4} \mathrm{~km}}\right) .
$$

In the last equality in (28) we used estimation (8). The shift is of the order of original size of the wave packet, $\Delta x \sim \sigma_{x}$, which is complementary to the relation (25).

Since separation of wave packets is partial the effect of averaging depends on the shape of the wave packet. So, measuring dependence of the depth of variations of signal on the base-line ( $\eta$, see Fig. 2) one can in principle restore the shape of the wave packet. (Loss of coherence is due to relative shift of the wave packets and the coherence condition is determined by the original size of the wave packet and not by the length of the packet after spread [18]). This is very rare situation; another one is realized for supernova neutrinos propagating in the Earth [18].

2. Enhanced effect for the core crossing trajectories. For trajectories with $\eta<0.58$ the depth of oscillations averaged over the neutrino energy is factor of $4-5$ larger than for the deepest trajectories in the mantle. Furthermore, $\Delta P$ is positive during some part of the oscillation period. Both effects are due to higher matter density in the core and interplay of oscillation effect in the core and mantle. Appearance of the positive Earth matter effect, $\Delta P>0$, is also related to that fact that the average values of $A_{e}$ in the core and in the mantle should be the same. The latter can be explained by the attenuation effect (see below): with strong averaging the core should not be seen. The only what one can observe is the average probability which is the same in the core and mantle.

3. Attenuation effect. Integration over the energy leads to lost of sensitivity to the remote structures of the density profile [9]. This is characterized by the attenuation length, $\lambda_{\text {att }}$, in such a way that structures at distances from a detector bigger than $\lambda_{\text {att }}$ are not resolved in the oscillation picture. To find the dependence of $\lambda_{\text {att }}$ on $\eta$ explicitly we take for $g(E)$ a Gaussian profile with the width $\Gamma_{\mathrm{Be}}$. (The use of exact line shape does not change this result substantially.) In this case the expression (24) 


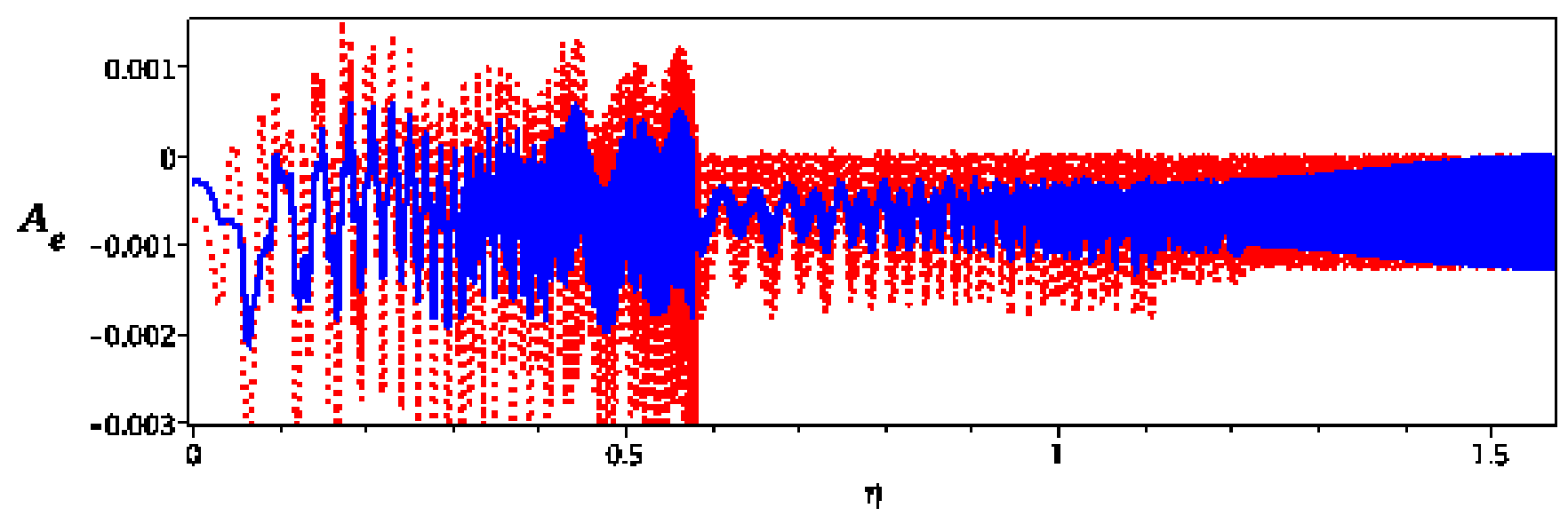

FIG. 1: The relative variations of the electron neutrino flux as function of the nadir angle of the neutrino trajectory. Dotted (red) line shows $A_{e}^{0}$ without averaging; solid (blue) line is $A_{e}$ which corresponds to the variations averaged over the energy spectrum of the ${ }^{7}$ Be neutrinos. Spherical symmetry of the Earth is assumed.
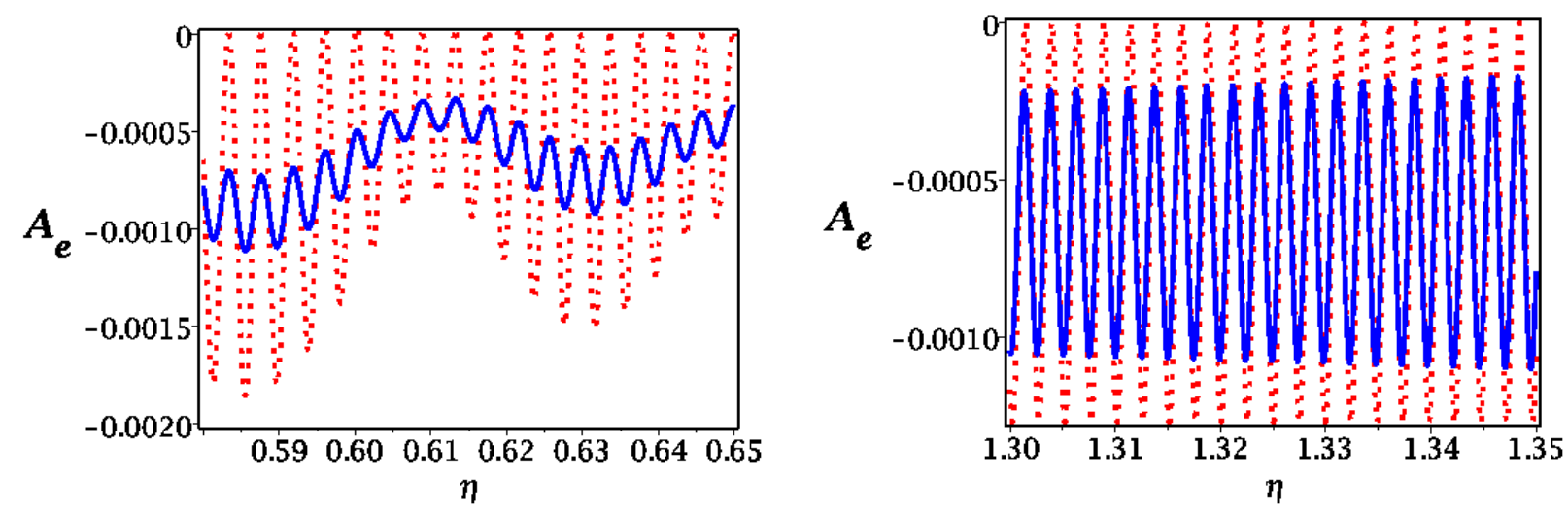

FIG. 2: The same as in Fig. 1 with two zoomed regions: $\eta=[0.58-65]$ (left) and [1.3 - 1.35] (right).

gives

$$
A_{e}=-\frac{1}{2} c_{13}^{2} f\left(\Delta m^{2}, \theta\right) \int_{0}^{L} d x V(x) F(L-x) \sin \phi_{x \rightarrow x_{f}}^{m},
$$

where

$$
F(L-x)=\exp \left\{-2\left[\frac{\pi \Gamma_{\mathrm{Be}}(L-x)}{E l_{\nu}}\right]^{2}\right\}
$$

According to Eqs. (29) and (30) a contribution to the integral (29) from structures situated at the distance $(L-$ $x)>\lambda_{\text {att }}$ from detector, where the attenuation length $\lambda_{\text {att }}$ is determined by the relation

$$
\sqrt{2} \frac{\pi \Gamma_{\mathrm{Be}} \lambda_{a t t}}{E l_{\nu}}=1
$$

becomes exponentially suppressed. This gives $\lambda_{\text {att }} \sim$ $7000 \mathrm{~km}$ which is comparable with radius of the Earth and bigger than the distance to the core. So, the core of the Earth should be seen in agreement with result of Fig. 1 (increase of the oscillation amplitude at $\eta=0.58$ ). The average values of oscillation curve in the mantle and the core are nearly the same.

4. Modulation of the oscillation picture; interference. At $\eta=1.21$ and $\eta=1.11$ the oscillatory picture changes: the depths of oscillations slightly increases, and high frequency oscillations start to be modulated by low frequency modes. Periods of modulations increase with decrease of $\eta$ (see Fig. 1). The modulations originate from the density jumps at distances $h_{1}=410 \mathrm{~km}$ (by 5\%) and at $h_{2}=660 \mathrm{~km}$ (by 10\%) from the surface of the Earth. These density changes occur over depth interval less than $5 \mathrm{~km}$ [19], [20], which is much smaller than the oscillation length. Therefore the jumps break adiabaticity. The adiabaticity is well satisfied within the layers bounded by the jumps. The jumps lead to increase of the depth of oscillations at $\sin \eta=1-h_{i} / R_{E}$. The periods of modulations in the $\eta$ scale can be obtained in the following 
way. The length of the trajectory with a given value of the nadir angle $\eta$ between the surface of the Earth and the $i$ th jump equals

$$
x_{i}(\eta)=R_{E} \cos \eta-\sqrt{R_{E}^{2} \cos ^{2} \eta-2 R_{E} h_{i}+h_{i}^{2}} .
$$

Consequently, the length of trajectory in the layer between the $\mathrm{i}$-th and $\mathrm{j}$-th jumps is $x_{i j}=x_{i}(\eta)-x_{j}(\eta)$. Then the period of modulations, $\delta(\eta)=\eta_{1}-\eta_{2}$ is determined by the condition

$$
\left[x_{i}\left(\eta_{1}\right)-x_{j}\left(\eta_{1}\right)\right]-\left[x_{i}\left(\eta_{2}\right)-x_{j}\left(\eta_{2}\right)\right]=l_{m} .
$$

In the approximation of flat layers $\left(R_{E} \rightarrow \infty\right)$ or $R_{E} \cos \eta \gg h_{i}$ the condition simplifies: the length of the trajectory equals approximately $h_{i} / \cos \eta$ and instead of (32) we obtain

$$
\Delta(\cos \eta)=\left(\cos \eta_{1}-\cos \eta_{2}\right) \approx \frac{l_{\nu}}{h_{i}} \cos ^{2} \eta .
$$

From $(32,33)$ for $\eta=0.58$ (the deepest mantle trajectory) we find $\Delta \eta=0.08$ in agreement with the results of Fig. 1. With increase of $\eta$ the period of modulations decreases. Thus, characteristics of modulations encode information about parameters of non-adiabatic density jumps (their position and size). Modulations become especially profound for the deepest mantle trajectories.

\section{Geophysics of the Be neutrino oscillations}

At the sub-percent level of the experimental accuracy and very short oscillation length a number of new effects become accessible and should be taken into account. Recall that the oscillatory curves shown in Figs. 1, 2 correspond to the ideal spherically symmetric Earth. In reality the Earth profile is not symmetric and deviations are of the order of oscillation length. Deviations include:

(i) Non-sphericity of the Earth as whole.

(ii) Small scale structures at the surface (mountains, oceans, etc.).

(iii) Structures in the crust (layers with anomalous density, cavities).

(iv) Possible structures in the mantle and the core, in particular deviation of the core from spherical form.

Effects of the deviations on $A_{N}$ can be quantified by contributions to the total oscillation phase $\Phi_{m}$ which can be written according to (15), (16) as

$$
\begin{aligned}
\Phi_{m} & =2 \pi \frac{L}{l_{\nu}}\left[1-\cos 2 \theta_{12} c_{13}^{2} \frac{1}{L} \int d x \epsilon(x)\right] \\
& =2 \pi \frac{L}{l_{\nu}}\left(1-\cos 2 \theta_{12} c_{13}^{2} \bar{\epsilon}\right) .
\end{aligned}
$$

Here $\bar{\epsilon}(L)$ is the averaged value of the matter parameter over the neutrino trajectory with the length $L$. The matter correction to $\Phi_{m}$ (last term in (34)) equals

$$
\Delta \Phi_{m}=2 \pi \frac{L}{l_{\nu}} \cos 2 \theta_{12} \bar{\epsilon}=\frac{\pi}{2}\left(\frac{\bar{\epsilon}}{10^{-3}}\right)\left(\frac{L}{7000 \mathrm{~km}}\right) .
$$

For $\bar{\eta}=1.2$ (the outer mantle) we obtain $\Delta \Phi_{m}=0.9 \pi$. For $\bar{\eta}=0.58$ (the deepest trajectory in the mantle) the corresponding number is $\Delta \Phi_{m}=3 \pi$. For $\bar{\eta}=0.4$ (the core crossing trajectory) we find $\Delta \Phi_{m}=5.9 \pi$. So, to avoid an uncertainty in the phase, $\bar{\epsilon}$ should be known with accuracy better than $20 \%, 8 \%$ and $4 \%$ in the outer mantle, deep mantle and core crossing trajectories. In general, all possible corrections and uncertainties (e.g. in $L, \Delta m_{21}^{2}$, etc.) which change the phase by $\sim \pi / 6$ should be taken into account. In view of many oscillation periods obtained over the baseline even small effects may become important. Large uncertainties in the density profile would wash out the oscillatory dependence.

Let us consider some effects of density profile perturbations.

1. Deviation of the Earth shape from ideal sphere can be characterized as follows. The polar and equatorial radii equal $R_{\text {pol }}=6356.7 \mathrm{~km}$ and $R_{e q}=6378.1 \mathrm{~km}$ correspondingly. The difference in diameters is about 43 $\mathrm{km}$. For a given latitude $\psi$ the distance from the surface to the center of the Earth can be approximated by

$R_{\psi} \approx R_{e q}-\left(R_{e q}-R_{p o l}\right) \cdot \sin ^{2} \psi=R_{e q}-21.5 \mathrm{~km} \cdot \sin ^{2} \psi$.

Introducing the average radius of the Earth as $\bar{R}=$ $0.5\left(R_{e q}+R_{p o l}\right)$ we obtain the deviation from the average $R_{\psi}-\bar{R}=\left(R_{e q}-R_{p o l}\right)\left(0.5-\sin ^{2} \psi\right)$. E.g. for equator $(\psi=0)$ the difference of trajectory lengths can be as large as $\left(R_{e q}-R_{p o l}\right) / 2=21.4 \mathrm{~km}$.

Due to non-sphericity of the Earth for the same value of the nadir angle the length of trajectory and consequently the oscillation phase depend on the azimuthal angle. Therefore deviation from sphericity leads to modification of the oscillatory curves in Figs. 1, 2. If variable $\eta$ is used, one needs to average phase over the azimuthal angle. This however, may lead to complete averaging of the oscillations. Indeed, for a detector at the latitude $\psi$ and for small nadir angle $\eta$ the difference of lengths of trajectories for different azimuthal angles can be as large as

$$
\delta x \approx 2 \bar{R}(r-1) \tan \eta \sin 2 \psi
$$

It can be about $(10-20) \mathrm{km}$ even for small $\eta$.

Inversely, due to non-sphericity the trajectories with the same $L$ have different $\eta$ and azimuthal angles and therefore $L$ does not fix the density profile uniquely. So $\bar{\epsilon}$ can be different for trajectories with the same $L$ but different $\eta$. This variation, however, can be neglected in the first approximation, and one can use $L$ as the parameter to mark events.

2. The effect of small structures at the surface of the Earth depends on $\eta$. Let us neglect here non-sphericity 
and use $\eta$ variable. Small structures produce distortion of the periodic sinusoidal curves shown in Figs. 1, 2 leading to appearance of irregular perturbations, substructures, shifts of maxima and minima, etc..

Consider perturbation produced by a structure at the point where neutrino enters the Earth (the beginning of the trajectory) which has the surface length $x_{\text {pert }}$ and height $h_{\text {pert }}$. Modification of $A_{e}(\eta)$ produced by this structure depends on relative values of $x_{\text {pert }}$ and the distance $x_{T}$ which corresponds to $\eta_{T}-$ period of $A_{e}(\eta)$ in $\eta$. In turn, $\eta_{T}$ is determined from the equation

$$
2 R_{E}\left[\cos \eta-\cos \left(\eta+\eta_{T}\right)\right]=l_{m} \approx l_{\nu}
$$

which can be rewritten as

$$
\sin \eta \sin \eta_{T}+\cos \eta\left(1-\cos \eta_{T}\right)=\frac{l_{m}}{2 R_{E}} .
$$

Then the distance $x_{T}$ as function of $\eta$ is given by

$$
x_{T} \approx \sqrt{l_{\nu}^{2}+\left[4 R_{E} \cos \eta \sin \left(0.5 \eta_{T}\right)\right]^{2}} .
$$

For very shallow trajectories $(\eta \sim 1.5), x_{T} \sim l_{\nu} \sim 28$ $\mathrm{km}$, which is comparable with the length of perturbations.

With decrease of $\eta$ the $x_{T}$ increases. For directions not very close to vertical the first term in (38) dominates and we obtain $\sin \eta_{T} \approx l_{m} /\left(2 R_{E} \sin \eta\right)$, or

$$
\eta_{T} \approx \frac{l_{m}}{2 R_{E} \sin \eta}
$$

Insertion of this $\eta_{T}$ into (39) gives

$$
x_{T} \approx \frac{l_{\nu}}{\sin \eta} .
$$

E.g. for the deepest trajectories in the mantle, $\sin \eta=$ 0.56 , we obtain $x_{T}=50 \mathrm{~km} . x_{T}$ increases faster near vertical directions. For $\eta=0$ we find from (38) and (39)

$$
x_{T} \approx 2 \sqrt{l_{\nu} R_{E}} \approx 850 \mathrm{~km}
$$

The size of perturbation of $A_{e}$ is given by the additional phase acquired due to perturbation of the profile:

$$
\Phi_{\text {pert }} \approx 2 \pi \frac{L_{\text {pert }}}{l_{\nu}}
$$

where $L_{\text {pert }} \approx h_{\text {pert }}$ for nearly vertical trajectories, and $L_{\text {pert }} \approx x_{\text {pert }}$ for nearly horizontal trajectories. The change of $A_{e}$ equals

$$
\Delta A_{e} \approx \frac{1}{2} c_{13}^{2} \epsilon f\left[\cos \Phi_{m}-\cos \left(\Phi_{m}+\Phi_{\text {pert }}\right)\right] .
$$

For shallow trajectories already $l_{\text {pert }} \approx x_{\text {pert }}=5 \mathrm{~km}$ become important. $\Phi_{\text {pert }}=0.35 \pi$ and it will change $A_{e}$ in the interval $\Delta \eta \sim x_{\text {pert }} / x_{T} \approx x_{\text {pert }} / l_{\nu}=0.17$ of the period. The changes of $A_{e}$ in units $A_{e}^{\max }=-c_{13}^{2} \epsilon f$ are
$0 \rightarrow 0.2,0.5 \rightarrow 0.95,1 \rightarrow 0.73$ for $\Phi_{m}=0, \pi / 2, \pi$ correspondingly. This also means that maximum and minimum will be shifted. For $x_{\text {pert }}=15 \mathrm{~km}$ the effect will be of the order 1, e.g. maximum will become minimum and vice versa. In this case the change can be viewed as a local shift of the oscillatory curve by half of period in $\eta$.

With decrease of $\eta$ effect of perturbation of the same size will decrease since $x_{T}$ becomes larger and $l_{\text {pert }}$ becomes smaller. That is, the change will be over smaller part of the period and the change of $A_{e}$ will be smaller.

For vertical trajectories we have $x_{\text {pert }} \ll x_{T}$ and $\Phi_{\text {pert }} \leq 0.35 \pi$. So, the effect shows up as small perturbations of oscillatory curve with typical size being much smaller than the period.

Since the oscillation length is about $30 \mathrm{~km}$, small structures at the surface of the Earth (mountains, oceans, seas) as well as in the crust may become important. Relatively small structures can be averaged out when integrating over the nadir angle intervals.

3. It is more difficult to control underground structures. Although variations of density in regions of $(5$ - 30) $\mathrm{km}$ size do not produce significant change of the phase and their effect will be subleading [9]. In principle one can study the shape of the core of the Earth and its deviation from sphere.

In this connection let us estimate effect of finite size of the ${ }^{7}$ Be neutrino production in the Sun. From the Earth the ${ }^{7} \mathrm{Be}$ neutrino production region in the Sun is seen as a disk of the angular size $\eta_{\text {disc }} \sim 10^{-4}$ radians. Neutrinos produced in different parts of the disc will have slightly different nadir angles and therefore baselines. Therefore one needs to perform integration over the corresponding intervals of the nadir angle. The value of $\eta_{\text {disc }}$ is an order of magnitude smaller than the oscillatory period $\Delta \eta \approx 0.002-0.003$, so the effect of averaging over disc is expected to be small.

To quantify this we approximate the distribution of the neutrino sources in the Sun by

$$
f\left(\eta^{\prime}, \eta\right)=\frac{1}{\delta_{\eta} \sqrt{2 \pi}} e^{-\frac{\left(\eta-\eta^{\prime}\right)^{2}}{2 \delta_{\eta}^{2}}}
$$

with $\delta_{\eta} \simeq 1.9 \times 10^{-4}$. The result shown in Fig. 3, indeed, confirms the smallness of the effect. Averaging over the disc is slightly stronger for large nadir angles where the oscillatory period is smaller.

\section{OBSERVATIONAL EFFECTS}

Observable effects consist of certain time variations of the signal during the nights, $A_{N}(t)$. Variations have 

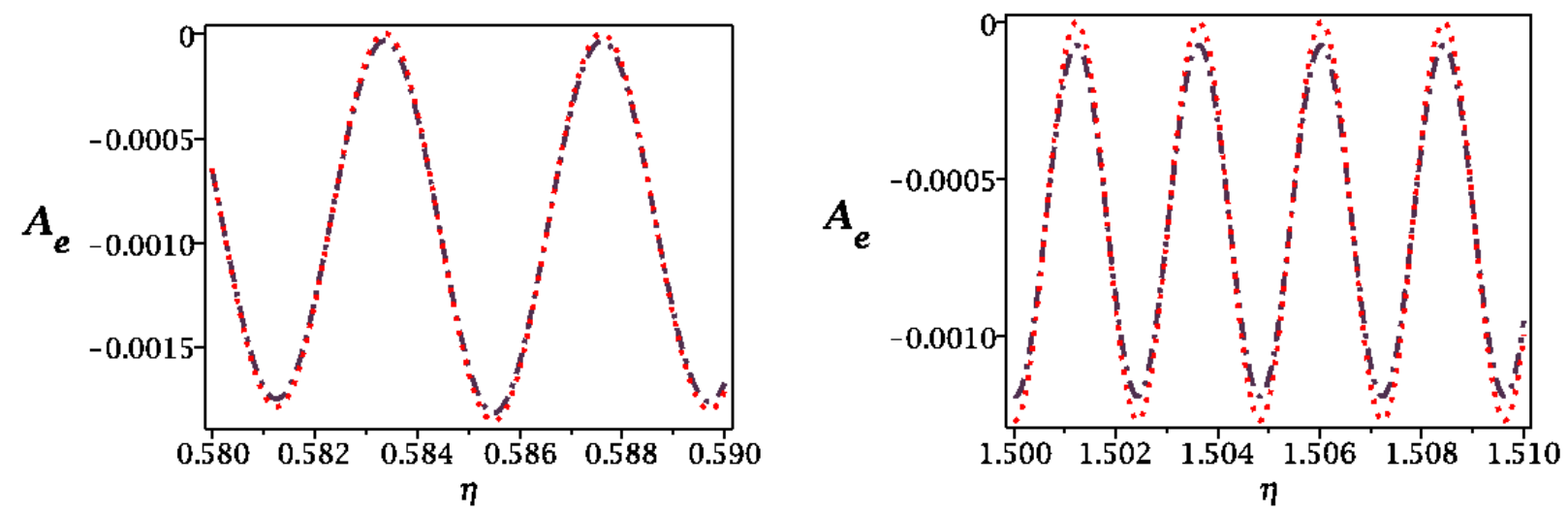

FIG. 3: Effect of averaging over the production region of ${ }^{7}$ Be neutrinos in the Sun. The relative change of the electron neutrino flux for mantle crossing trajectories with $\eta=0.58 \ldots 0.59$ (left) and $1.5 \ldots 1.51$ (right) without (dotted line) and with (dash-dotted line) averaging.

quasiperiodic dependence on time, the amplitude of variations also changes during the night, furthermore oscillations with high frequency are modulated by lower frequencies. Sinusoidal dependence is modified by nonsphericity of the Earth, small structures of the profile, etc.. Parameters of these variations depend on position of a detector.

In our estimations of sensitivities to different effects we will use for simplicity the spherical Earth. Deviations from sphericity should be taken into account for different detectors individually. As function of $L$ the oscillatory curve has regular dependence which is similar to the dependence on $\cos \eta$ for spherical case. Local deviations from sphericity produce complicated effects and perturbations of the profile should be taken into account.

In the case of spherical Earth and without small structures we can use $z \equiv \cos \eta$ as variable and evaluate number of events in small $\Delta z$ bins. Recall that different detectors cover the nadir angle ranges with minimal $\eta_{\text {min }}$ which depends on the latitude of the detector.

\section{Rate of events in the detectors}

The rate of $\nu-e$ scattering events in a detector can be written as

$R(t)=N_{e} \int d E F_{B e}(E, t) \sigma_{e}(E)\left[P(E, t)+r_{N C}(1-P(E, t))\right]$,

where $N_{e}$ is the total number of electrons in a fiducial volume, $\sigma_{e}$ is the cross-section of $\nu_{e}-e$ scattering, $r_{N C} \equiv \sigma_{N C} / \sigma_{e}$, and $\sigma_{N C}$ is the cross-section of the $\nu_{\mu}-e$ scattering due to neutral currents. The last term in (44) accounts for the contribution of the $\nu_{\mu}$ and $\nu_{\tau}$ neutrinos that are generated by oscillations. In the spherical Earth approximation dependence of the probability on time is via $\eta(t)$ only: $P(E, t)=P(E, \eta(t))$ The rate (44) can be rewritten as

$R_{N}(t)=N_{e} F_{B e}^{0}(t) \bar{\sigma}_{e} \int d E g(E)\left[P(E, t)\left(1-r_{N C}\right)+r_{N C}\right]$,

where $\bar{\sigma}$ is the averaged (over the ${ }^{7} \mathrm{Be}$ line) value of crosssection.

Let us introduce the relative variations of the rate of events due to the Earth matter effect

$$
A_{N}(t)=\frac{R_{N}(t)-R_{D}(t)}{R_{D}(t)} .
$$

Here $R_{D}(t)$ is the rate which would be without Earth matter effect and it is given by Eq. (45) with $P_{N}$ substituted by $P_{D}$. Inserting (45) into Eq. (46) and assuming that $\theta_{12}^{m}$, and consequently, $P_{D}$ do not change with energy in the interval of energies of the ${ }^{7} \mathrm{Be}$ neutrinos, we obtain

$$
A_{N}(t)=A_{e}(t) \kappa
$$

where

$$
\kappa \equiv \frac{1-r_{N C}}{1+r_{N C}\left(1 / P_{D}-1\right)}
$$

gives the correction due to contribution of $\nu_{\mu}$ and $\nu_{\tau}$. For $r_{N C}=0.2$ and $P_{D} \approx 0.6$ we obtain $\kappa=0.7$. Thus, the correction leads to damping of variations of signal.

For estimations we will consider future scintillator (or scintillator uploaded) detector with fiducial mass $M_{D}=$ 100 kton and 5 years exposure as illustrative values. By simple re-scaling one can find effects in specific proposed detectors such as LENA, JUNO or WBLS.

To evaluate the total number of events in such a detector we will use the rate estimated in [26] (see also [25]): 
$1.5 \cdot 10^{4}$ events per day in $48 \mathrm{kt}$. Normalizing to this number we obtain

$$
N^{t o t}=5.7 \cdot 10^{7}\left(\frac{M_{D}}{100 \mathrm{kton}}\right)\left(\frac{t}{5 \text { years }}\right) \text { events. }
$$

These events can be analyzed in various ways to make the data sensitive to different quantities.

\section{Establishing the Earth matter effect}

For this it is enough to determine value $A_{N}$ averaged over $\eta$. So, one should compare the total numbers of events detected during the nights and days during whole the exposure. (Possible distortion of $A_{N}(t)$ by perturbations of the density profile is not relevant here.) We take that approximately half of the total number of events (49) is detected during nights and another half during days:

$$
N_{N} \approx N_{D}=2.85 \cdot 10^{7} \text { events. }
$$

Then difference of the numbers of night and day events due to oscillations in the Earth equals

$$
N_{D}-N_{N} \approx-N_{D} A_{N}=-N_{D} \kappa A_{e}=1.7 \cdot 10^{4} \text { events. }
$$

The statistical error of measurements of $N_{D}-N_{N}$ is $\sigma_{N}=$ $\sqrt{2 N_{N}}=7.5 \cdot 10^{3}$. So, after 5 years

$$
\left(N_{D}-N_{N}\right) \approx 2.3 \sigma_{N} .
$$

That is, the difference of the night and day signals can be established at $2.3 \sigma$ level. This is in agreement with estimation made in the introduction. Notice that various systematic uncertainties cancel in the relative variations.

\section{Variations of the signal during nights}

One can measure oscillatory variations of the ${ }^{7} \mathrm{Be}$ neutrino signal during nights (Fig. 1) detecting events in short time intervals. This method may have less systematics than the first one described in the previous subsection. However here new systematics may appear due to unaccounted effects of small structures of the Earth (see below). Period of time variations can be estimated for spherically symmetric profile in the following way. The period in the nadir angle scale is given in (40), $\eta_{T}=2.24 \cdot 10^{-3} / \sin \eta$. Then the period in time equals

$$
t_{T}=\frac{l_{m}}{2 R_{E} \sin \eta}\left(\frac{d \eta}{d t}\right)^{-1}
$$

where the speed of change of the $\eta$ with time depends on value of $\eta$. We find that the average period equals about $1 \mathrm{~min}$. So, to measure the oscillatory curve one needs to take time intervals smaller than 15 sec. Number of events expected in such an interval will be about 3 5. Therefore summation of signals from time intervals in which the Earth matter effect is the same during several years is needed.

In the approximation of spherically symmetric Earth the length of trajectory and the density profile are fixed uniquely by the nadir angle $\eta$, and the Earth matter effect is quasi-periodic function of $\cos \eta$ (since $L=2 R_{E} \cos \eta$ ). Then from (34) we obtain the period in $\cos \eta$

$$
(\cos \eta)_{T}=\frac{l_{\nu}}{2 R_{E}\left(1-\cos 2 \theta_{12} c_{13}^{2} \bar{\epsilon}\right)} .
$$

Due to increase of $\epsilon(\eta)$ with decrease of $\eta$ the period will slightly increase for deeper trajectories. The relative change of period is of the order $10^{-3}$. It would be exactly periodic function in the case of constant density.

So, the method consists of splitting the whole $\cos \eta$ interval for a given detector into small intervals $\Delta(\cos \eta)<$ $(\cos \eta)_{T}$, identification the corresponding time intervals during nights, $\Delta t=\Delta t(\cos \eta, \Delta(\cos \eta))$, and accumulation of events in these small intervals during several years.

However, even with 100 kton detector it is not possible to measure whole the oscillatory curve of Fig. 1 with appreciable statistical significance. Therefore one should also sum up the signal over all periods of Fig. 1. Introducing corrections due to change of period with $\eta$ one can combine all the events in a single period using the effective phase (34) as variable. This means that for each event or several events detected during small enough time intervals $\Delta t=(10-15) \mathrm{sec}$, one finds $\Phi_{m}(t)$, and then collects events for the intervals $\Phi_{m}(t)+2 \pi k$.

Following this we divide all the events detected during the nights in to two groups: events detected in the first half of period, $N_{1}$, and events detected in the second half of period, $N_{2}$. Then difference of the events equals

$$
N_{1}-N_{2}=\langle D\rangle N_{N} \approx\langle D\rangle N_{D},
$$

where

$$
\langle D\rangle=\frac{2}{\pi}\left(A_{N}^{\max }-A_{N}^{\min }\right) .
$$

Here $A_{N}^{\max }$ and $A_{N}^{\min }$ maximal and minimal values of $A_{N}$ (averaged over different periods) and factor $2 / \pi$ reflects decrease of the difference due to integration over half periods as compared to the total depth. Thus, $\langle D\rangle$ is the relative variation of number of events integrated over half a period. From Fig. 1 we find that for the mantle trajectories $\kappa\left(A_{N}^{\max }-A_{N}^{\min }\right) \approx 5.2 \cdot 10^{-4}$. Taking $N_{N} \approx 2.8 \cdot 10^{7}$, we obtain $N_{1}-N_{2}=0.9 \cdot 10^{4}$. The statistical error of measurements of the difference $\left(N_{1}-N_{2}\right)$ is $\sqrt{N}_{N}=5.3 \cdot 10^{3}$. Correspondingly, variations during the night can be established at $1.8 \sigma$ level.

Let us estimate effect from the core crossing trajectories. We assume that fraction of events collected from 


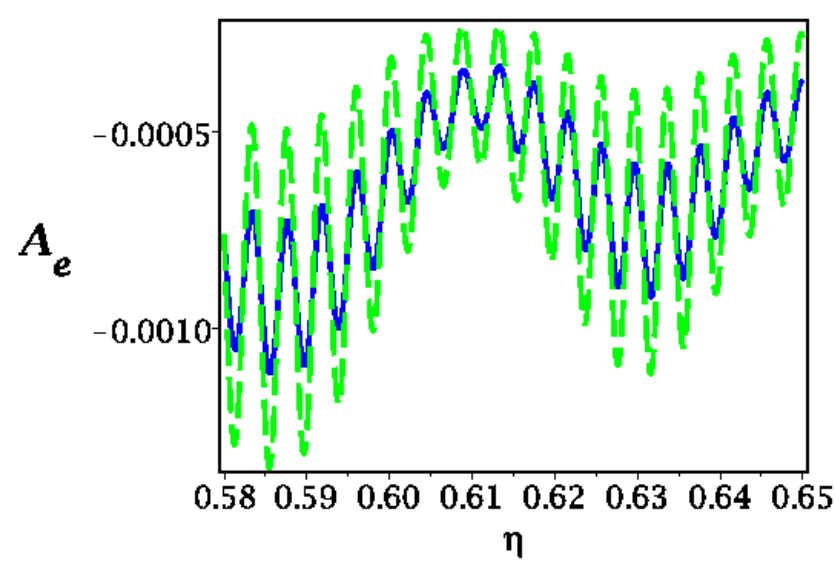

FIG. 4: The relative change of the electron neutrino flux for the mantle crossing trajectories as the function of $\eta$ for two different values of width of the ${ }^{7} \mathrm{Be}$ line which correspond to two different temperatures in the center of the Sun: Solid line for sun central temperature $T_{\odot}=15.55 \times 10^{6}$ (solid line) $7.77 \times 10^{6}$ degree (dashed line).

these trajectories is about $10 \%$, that is, $N_{\text {core }}=2.8 \cdot 10^{6}$. The depth of modulation is larger: $A_{N}^{\max }-A_{N}^{\min }=$ $2 \cdot 10^{-3}$. The expected difference of events in the first and second halves of the period equals $N_{1}-N_{2}=2.5 \cdot 10^{3}$. The statistical errors is $\sigma=\sqrt{N_{D}}=1.7 \cdot 10^{3}$. Therefore $N_{1}-N_{2}=1.5 \sigma$. Summing up the significances in the core and mantle we obtain that variation of the signal can be established at $2.3 \sigma$ level. These estimations have been performed for the ideal periodic oscillatory dependence of $A_{N}$ on $\cos \eta$. As we discussed in Sec. 3, the presence of small structures at the surface of the Earth and in the crust distorts the sinusoidal dependence, in particular it can shift significantly maxima and minima in the $\cos \eta$ scale. This leads to uncertainties in determination of borders of periods and intervals in which events should be summed up. The uncertainties can be reduced if a profile of the Earth along trajectory of each event is known and therefore for each event the oscillation phase can be determined. Actually, it will be enough to know the length of trajectory, and the rest can be accounted as systematic error. As a result, significance of establishing of variations may be somehow lower.

\section{Determination of the line width}

The depth of oscillations in the Earth decreases with increase of the width of the line which in turn is proportional to the central temperature in the Sun: $\Gamma_{\mathrm{Be}} \propto T_{c}$. In Fig. 4 we show the oscillatory picture for two different widths of the line which correspond to two different central temperatures of the Sun. As follows from Fig. 4, the depth of oscillations increases by factor 2 with decrease of the temperature by the same factor.
According to Fig. 1 for shallow trajectories, $\eta>1.4$, the averaging effect is negligible and the depth of oscillation is the same as for monochromatic spectrum. The averaging effect strengthens and depth decreases with decrease of $\eta$. For larger width $\Gamma_{\mathrm{Be}}$, the change of depth is faster. The depth of oscillations is larger for the corecrossing trajectories and it changes with $\eta$ weakly. Therefore methods of determination of the width can be based on determination of the depth of variations $A_{N}$ and its dependence of the depth on $\eta$. One can

1. measure the average depth of variations and compare it with expectations;

2. compare the average depths for deep (e.g. $\eta=$ $0.58-1.20)$ and outer $(\eta=1.20-1.57)$ trajectories in the mantle;

3. compare of the average depths in the core and in the mantle.

Let us consider the first method. According to Fig. 4 the depth $\langle D\rangle \propto \frac{1}{T}$ and $D(\eta)$ changes linearly with $\eta$ (see Fig. 1). Therefore we can write for the averaged depth for all mantle trajectories

$$
\langle D\rangle=\frac{2}{\pi}\left(0.63+0.19 \frac{T_{c}^{s t}}{T_{c}}\right) \cdot 10^{-3},
$$

where $T_{c}^{s t}$ is the standard value of temperature in the center of the Sun. The first term in the brackets corresponds to the half depth of oscillations at the surface (trajectories with large $\eta$ ), the second one corresponds to the deepest trajectories in the mantle for $T_{c}=T_{c}^{s t}$. For $T_{c}=T_{c}^{s t}$ we obtain from $(57)\langle D\rangle=0.51 \cdot 10^{-3}$, whereas for $T_{c}=0.5 T_{c}^{s t}:\langle D\rangle=0.64 \cdot 10^{-3}$. So, decrease of the $T_{c}$ by factor 2 leads to increase of the average depth by $20 \%$. As we have found in the previous subsection the deviation of the average depth from 0 can be established with significance about $2.5 \sigma$, so its $20 \%$ change would correspond to about $0.5 \sigma$.

Consider the second method. According to Fig. 1, the average depth in the outer part of mantle $(\eta=1.20-$ 1.57), equals $\langle D\rangle=0.64 \cdot 10^{-3}$, whereas in the inner part $(\eta=0.58-1.20):\langle D\rangle=0.33 \cdot 10^{-3}$. We take that the number of events in the inner and outer intervals of $\eta$ are the same and equal $N_{N} / 2$, so that variations in the outer and inner ranges of $\eta$ equal $6.7 \cdot 10^{3}$ and $3.4 \cdot 10^{3}$ correspondingly. The statistical error is $3.9 \cdot 10^{3}$, therefore variations can be established at $1.7 \sigma$ (outer) and $0.9 \sigma$ (inner). The ratio of the depths (which is sensitive to temperature) equals

$$
\frac{\langle D\rangle_{\text {in }}}{\langle D\rangle_{\text {out }}}=0.51 \pm 0.66
$$

where the $1 \sigma$ statistical errors is indicated. So, even decrease of the ratio by factor 2 will correspond to $0.4 \sigma$.

Sensitivity is low but this is independent measurement of the width. Clearly, factor of 2 uncertainty in central temperature is excluded, e.g., by measurements of the boron neutrino flux. 


\section{Determination of $\Delta m_{21}^{2}$}

The oscillatory pattern of Fig. 1 depends on precise value of $\Delta m_{21}^{2}$. Uncertainty in $\Delta m_{21}^{2}, \delta\left(\Delta m_{21}^{2}\right)$, would not influence the pattern if

$$
\frac{\delta\left(\Delta m_{21}^{2}\right)}{\Delta m_{21}^{2}} \ll \frac{l_{\nu}}{L_{\max }} \approx 0.3 \% .
$$

The present accuracy of determination of $\Delta m_{21}^{2}$ is $2.5 \%$ and future experiments (e.g. JUNO-reactors) will not be able to reach $0.1 \%$ accuracy. So, $\Delta m_{21}^{2}$ should be extracted from the ${ }^{7}$ Be-neutrino studies simultaneously with other measurements.

The mass splitting $\Delta m_{21}^{2}$ determines the depth, the average value of probability and the length of oscillations. Correspondingly, there are several different methods to measure $\Delta m_{21}^{2}$. The depth of variations of $A_{N}$ and its average value according to Eqs. (17) and (21) are inversely proportional to the splitting: $D \propto 1 / \Delta m_{21}^{2}$, $A_{e} \propto 1 / \Delta m_{21}^{2}$. As we have established in the previous subsection, $A_{e}$ and $D$ can be distinguished from 0 at about $2.3 \sigma$ level. Consequently, these measurements will determine $\Delta m_{21}^{2}$ with about $1 / 2.3 \approx 40 \%$ accuracy at $1 \sigma$ level.

Since there are many (up to 400, according to Fig. 1) periods of oscillations, dependence of the oscillation period on $\Delta m_{21}^{2}$ can provide much better determination of $\Delta m_{21}^{2}$. Indeed, the error $\delta\left(\Delta m_{21}^{2}\right) \sim(1 / 300) \Delta m_{21}^{2}$ would lead to substantial change of the oscillatory picture. Clearly knowledge of exact values of $L$ for the detected events is crucial.

The analysis can be performed using, e.g., the LombScargle [27], [28] method which is valid for uneven time intervals. Let

$$
N_{N}^{j}=N_{D}^{j}\left(1+A_{N}^{j}\right)
$$

be the observed number of events in the $j$ - bin with the average nadir angle $\eta_{j}$. This corresponds to the true value of $\Delta m_{21}^{2}=\Delta m_{21}^{\text {true }} 2$. The first term in (59) can be considered as fluctuating noise, whereas the second one as periodic signal to be extracted. For constant density we can use expression (21), so that

$$
N_{N}^{j} \approx N_{D}^{j}\left(1+\frac{1}{2} c_{13}^{2} \epsilon f \cos \Delta_{m}^{t r u e} L_{j}\right)
$$

The method require that the mean value of the background is zero. So, we need to subtract the average day signal $\bar{N}_{D}$ from $N_{N}^{j}$. Then the Lomb-Scargle periodogram is defined as

$$
P_{L S}=\frac{1}{n} \frac{\left(\sum_{j}\left[N_{N}^{j}\left(\Delta_{m}^{\text {true }}\right)-\bar{N}_{D}\right] \cos \Delta_{m}^{f i t} x_{j}\right)^{2}}{\sum_{j} \cos ^{2} \Delta_{m}^{f i t} x_{j}}
$$

where $n$ is the total number of bins and $\Delta_{m}^{f i t}$ corresponds to the fit value of $\Delta m_{21}^{2}$. The true value of $\Delta m_{21}^{2}$ can be obtained by varying $P_{L S}$ over $\Delta m_{21}^{\text {fit } 2}$ : it corresponds to maximum of $P_{L S}$.

For illustration we can perform simplified computations using constant density profile and spherically symmetric Earth. In this case the $N_{N}$ is exactly periodic function of $\cos \eta$.

The Lomb-Scargle periodogram is based on the discrete Fourier transform, but in the case of many bins of even size we can substitute summation in eq. (61) by integration over $x \propto \cos \eta$ from 0 to $x_{\max }$ (here $x$ is the length of the trajectory). So,

$$
P_{L S}=\frac{\left[x_{\max }^{-1} \int d x\left[N_{N}(x)-\bar{N}_{D}\right] \cos \Delta_{m}^{f i t} x\right]^{2}}{n x_{\max }^{-1} \int d x \cos ^{2} \Delta_{m}^{f i t} x}
$$

where

$$
N_{N}(x) \approx N_{D}(x)\left(1+\frac{1}{2} c_{13}^{2} \epsilon f \cos \Delta_{m}^{\text {true }} x\right) .
$$

The integration in (62) gives

$$
P_{L S}=N_{D}^{2} \frac{\left(c_{13}^{2} \epsilon f\right)^{2}}{8}\left|\frac{\sin \Delta \Phi}{\Delta \Phi}\right|^{2},
$$

where

$$
\Delta \Phi=\left(\Delta_{m}^{\text {true }}-\Delta_{m}^{f i t}\right) x_{\max }
$$

Using expression for $\Delta_{m}$ in vacuum we find the following relation for $\delta\left(\Delta m_{21}^{2}\right) \equiv \Delta m_{21}^{\text {true }}{ }^{2}-\Delta m_{21}^{\text {fit } 2}$ :

$$
\frac{\delta\left(\Delta m_{21}^{2}\right)}{\Delta m_{21}^{2}}=\frac{\Delta \Phi}{4 \pi} \frac{l_{\nu}}{x_{\max }}
$$

For $\Delta \Phi=\pi / 2$, which corresponds to half of the height of the peak in (64), Eq. (66) gives

$$
\frac{\delta\left(\Delta m_{21}^{2}\right)}{\Delta m_{21}^{2}}=4 \cdot 10^{-4}
$$

These estimations do not take into account effect of averaging over the ${ }^{7}$ Be-neutrino spectrum. So, realistic accuracy will be above 0.001 .

\section{Tomography of the Earth}

As we discussed in Sec. 3, the oscillatory pattern encodes information on the shape and density profile of the Earth. So, in principle one can perform tomography of the Earth with spatial precision comparable with the oscillation length. Let us summarize some dependences:

- Sudden change of the depth of variations of $A_{N}$ at $\eta \sim 0.58$ marks trajectories which start to cross the core. The corresponding $\eta$ gives the position of the density jump between the mantle and the core, the size of change of the depth of oscillations reflects the size of the jump. 
- Detailed oscillation pattern in the core region is sensitive to the parameters of the inner core.

- Small density jumps in the mantle trajectory range and modulations of the high frequency oscillations encode information about the mantle structure: position of borders between different regions (inner mantle, outer mantle, etc.) and sizes of density jumps at their borders.

- The oscillatory picture is sensitive to the shape of the Earth, in particular, to its deviation from sphericity.

- Distortion of the periodic oscillatory curve is sensitive to local structures (mountains, seas, oceans, oil layers, etc.) [11].

Detectors with $0.1 \%$ accuracy of measurements will see (resolve) core and mantle. Other features will have lower significance and appear as systematic errors in these detectors.

\section{SEARCHES FOR STERILE NEUTRINOS}

The parameter $\epsilon$, and consequently, the Earth matter effect are inversely proportional to the mass splitting $\Delta m_{21}^{2}$. Therefore with decrease of $\Delta m_{21}^{2}$ (for the same value of mixing) the variation $A_{N}$ increases. In this connection let us consider sterile neutrinos with very small mass splitting, $\Delta m_{10}^{2} \equiv m_{0}^{2}-m_{1}^{2} \ll \Delta m_{21}^{2}$. Here $m_{0}$ is the mass of new state. The matter parameter for the $\nu_{e}-\nu_{s}$ system, $\epsilon_{s}$, is determined by the potential $V_{s} \approx 0.5 V_{e}$. Then the resonance, $\epsilon_{s}=1$, is achieved for

$$
\Delta m_{10}^{2}=\Delta m_{10 R}^{2}=0.9 \cdot 10^{-7} \mathrm{eV}^{2} .
$$

(for the mantle densities). In the core the resonance enhancement will be at about 2 time bigger splitting.

For definiteness we will consider mixing of sterile neutrino in the mass state $\nu_{1}$ characterized by the angle $\theta_{s}$. In this case we obtain for the difference of the night and day signals

$$
\Delta P \approx\left|U_{e 1}\right|^{2}\left(\left|A_{01}\right|^{2}-\left|A_{00}\right|^{2}\right)\left(P_{1 e}^{s}-\left|U_{e 1}\right|^{2}\right),
$$

where $A_{01}$ and $A_{00}$ are the amplitudes of transitions between the matter eigenstates: $\nu_{0 m} \rightarrow \nu_{1 m}=\nu_{1}$ and $\nu_{0 m} \rightarrow \nu_{0 m}=\nu_{0}$ when neutrinos propagate from the center to the surface of the Sun. In (69) we have taken into account that in the Sun, for small mass splitting the neutrinos are produced far above $\nu_{1}-\nu_{0}$ resonance and in initial state $\nu_{0 m} \approx \nu_{1}$ which gives the factor $\left|U_{e 1}\right|^{2}$ in Eq. (69). Eq. (69) is analogy of expression (10).

Even for splitting (68) the coherence will be lost due to separation of the wave packets of the mass states on the way to the Earth. So, as in the active neutrino case, independent fluxes of $\nu_{1}$ and $\nu_{0}$ will arrive at the Earth surface.

For oscillations in the Earth we consider the $2 \nu$-system $\nu_{1}-\nu_{0}$ and denote by $P_{1 e}^{s}$ the probability of $\nu_{1} \rightarrow \nu_{e}$ transition. For estimations we use the constant density profile for the trajectories inside the Earth with average value of density. This is justified since due to smallness of $\Delta m_{21}^{2}$ the oscillation length is much larger than the size of the Earth. In this case

$$
P_{1 e}^{s}-\left|U_{e 1}\right|^{2}=-\epsilon_{s} \sin ^{2} 2 \theta_{s}^{m} \sin ^{2}\left(\frac{1}{2} \Delta_{m} L\right),
$$

For relatively large mixing angle the adiabatic transition $\nu_{0 m} \rightarrow \nu_{0}$ inside the Sun would lead to substantial additional suppression of the $\nu_{e}{ }^{7} \mathrm{Be}$-neutrino flux. Theoretical accuracy of the flux is about $1.4 \%$. Experimental accuracy (presently 5\%) will be much better. So, the survival probability should be larger than 0.98 , that is, the adiabaticity should be strongly broken with $\left|A_{01}\right|^{2}>$ 0.98. Using results for active neutrinos (see e.g. [22]) we estimate that this can be achieved if $\sin ^{2} 2 \theta_{s}<0.01$ for splitting in (68). Taking $\left|A_{01}\right|^{2}-\left|A_{00}\right|^{2} \approx 0.96$, $\left|U_{e 1}\right|^{2} \approx 0.67$ and $P_{D} \approx 5 / 9$, we obtain from Eqs. (69) and (70)

$$
A_{N} \approx-1.15 \epsilon_{s} \sin ^{2} 2 \theta_{s}^{m} \sin ^{2}\left(\frac{1}{2} \Delta_{m} L\right) .
$$

Let us consider maximal allowed value $\sin ^{2} 2 \theta_{s}=0.01$. In this case the width of the MSW resonance, $\Delta E=$ $2 \tan 2 \theta_{s} E \approx 0.2 E=190 \mathrm{kev}$, is much larger than the width of the line. Changing $\Delta m_{10}^{2}$ within $(20-40) \%$ would put the line at different points of the MSW resonance peak.

In the resonance we have $\epsilon_{s} \approx \sin ^{2} 2 \theta_{s m} \approx 1$ and therefore

$$
A_{e}^{r e s} \approx-1.15 \sin ^{2} \frac{1}{2} \Delta_{m} L(\eta) .
$$

For the resonance value of $\Delta m_{21}^{2}$ (68) the oscillation length in vacuum equals $l_{\nu}=2.5 \cdot 10^{4} \mathrm{~km}$ and in matter (in resonance) $l_{m}=l_{\nu} / \sin 2 \theta_{s}=2.5 \cdot 10^{5} \mathrm{~km}$, i.e. much larger than the diameter of the Earth. For the deepest trajectory in the mantle, $L \approx 10^{4} \mathrm{~km}$, the phase equals 0.126 rad., and according to Eq. (72) $A_{e}=0.017$. This is an order of magnitude larger than the effect for active neutrinos and of the order of the present accuracy. For $\cos \eta=0.4$ (middle trajectory in the mantle) we find $A_{e}=0.0045$. The effect monotonously increases as $\propto 1 /(\cos \eta)^{2}$, reaching maximum for the deepest trajectory (middle of the night) and then it decreases down to zero.

For the core crossing trajectories the effect can be more complicated. The MSW resonance in the core leads to appearance of another peak, and the ${ }^{7} \mathrm{Be}$ neutrino line can be in one peak or another. Also the interplay of the effects in the mantle and the core may occur.

If $\Delta m_{10}^{2}$ is more than $40 \%$ larger than the resonance value, the values of oscillation parameters become close 
to vacuum values: $l_{m} \approx l_{\nu}, \sin ^{2} 2 \theta_{s m} \approx 0.01$, so that

$$
A_{e} \approx-0.015 \epsilon_{s} \frac{\sin ^{2} 2 \theta_{s}}{10^{-2}} \sin ^{2} \frac{1}{2} \Delta_{m} L(\eta) .
$$

In this case still $\epsilon_{s}=O(1)$, and the oscillatory factor can be of the order 1 . Thus, the effect for the deepest trajectories in the mantle may reach $1 \%$. Since the oscillation length becomes comparable with the size of the Earth, the time dependence of the effect is more complicated, e.g. with two maxima symmetrically shifted from the middle of the night.

With further increase of $\Delta m_{10}^{2}$ (outside the resonance region) the size of the effect decreases as $1 / \Delta m_{10}^{2}$, and time dependence will acquire an oscillatory form with increasing number of periods.

Let us make similar estimation for $\sin ^{2} 2 \theta_{s}=0.001$. In resonance the oscillation length equals $l_{m}=8.5 \cdot 10^{5} \mathrm{~km}$ (about 3 times bigger than in the previous case). Correspondingly, the phases will be 3 times smaller, and the oscillatory factor will be an order of magnitude smaller As a result, for the deepest mantle trajectory $A_{e} \sim 10^{-3}$. Outside the resonance peak we obtain $A_{e}<0.001$. Thus, the effect decreases as $\sin ^{2} 2 \theta_{s}$ everywhere.

\section{CONCLUSIONS}

We explored in detail effects of propagation of the solar ${ }^{7}$ Be neutrinos in the matter of the Earth. We estimated a possibility to detect these effects with future large scintillator (or scintillator uploaded) detectors which will have $0.1 \%$ accuracy of measurements.

The main features of the propagation are determined by low energy of the ${ }^{7}$ Be neutrinos and their narrow energy spectrum. Oscillations in the Earth are pure matter effect and they are related to transitions between the mass eigenstates.

Physics of oscillations is determined by two accidental coincidences. Due to low energies, the oscillation length, $\approx 30 \mathrm{~km}$, is of the order of small structures of the Earth profile and non- sphericity of the Earth. The width of the spectrum is comparable with the period of the oscillatory curve in the energy scale. So that depending on length of trajectory (nadir angle) one should observe different degree of averaging. In configuration space this is equivalent to partial loss of coherence due to shift of the wave packets of different eigenstates of the Hamiltonian in the course of propagation inside the Earth. The size of the packets due to spread on the way from the Sun is several orders of magnitude larger than the shift.

The main observable is the oscillatory variation (mainly suppression) of the signal in time (with nadir angle) during the night. The depth of oscillations changes with time since the averaging of oscillations becomes stronger with increase of the length of trajectory. The depth is the largest for shallow trajectories and it is the smallest for the deepest mantle trajectories. It sharply increases when trajectory crosses the core. Small density jumps in the mantle and the core produce modulations of the oscillatory curve.

We find that for illustrative configuration of experiment (100 kt, 5 years of exposure) the Earth matter effect can be established at $2.3 \sigma$ level; the width of the ${ }^{7}$ Be-neutrino line can be determined with factor of 2 accuracy at $0.5 \sigma ; \Delta m_{21}^{2}$ can be measured with accuracy $0.1 \%$. The presence of the core of the Earth and its border can be seen by the detector (close to equator) at $2 \sigma$. The sensitivity can be enhanced if one uses larger exposure time (e.g. 10 years) or larger fiducial volume of the detector.

Determination of other characteristics: size of region in the Sun where the Be neutrinos are produced, detailed tomography of the Earth (small scale structures, layers in the mantle and the core) will require further substantial increase of the detector size.

At this level of accuracy it is not possible to measure whole oscillatory curve which contains interesting information about structure of the Earth. That would open up a possibility to use the Sun as the scanner of the Earth to perform tomography. However, estimations show that the required sensitivity is not by several orders of magnitude higher.

One can perform searches of sterile neutrinos with mixing $\sin ^{2} 2 \theta_{s}=10^{-3}-10^{-2}$ in wide range of $\Delta m_{10}^{2}>10^{-8}$ $\mathrm{eV}^{2}$, especially in the resonance region of mass splitting around $10^{-7} \mathrm{eV}^{2}$.

\section{Acknowledgements}

Ara Ioannisiyan thanks ICTP for visit in 2014, when part of this work has been accomplished.

\section{References}

[1] J. N. Bahcall, Phys. Rev. Lett. 71, 2369 (1993) J. N. Bahcall, Phys. Rev. D 49, 3923 (1994)

[2] according to recent measurements $E=861.81$ http://www.nucleide.org/DDEP WG/Nuclides/Be-7 tables.pdf and references therein.

[3] G. Bellini et al. [Borexino Collaboration], Phys. Rev. D 89, 112007 (2014) [arXiv:1308.0443 [hep-ex]].

[4] O. Smirnov et al. [Borexino Collaboration], arXiv:1410.0779 [physics.ins-det].

[5] A. Gando et al. [KamLAND Collaboration], arXiv:1405.6190 [hep-ex].

[6] G. Bellini et al. [Borexino Collaboration], Phys. Lett. B 707 (2012) 22 [arXiv:1104.2150 [hep-ex]]. 
[7] A. de Gouvea, A. Friedland and H. Murayama, JHEP 0103, 009 (2001) [hep-ph/9910286].

[8] J. N. Bahcall, M. C. Gonzalez-Garcia and C. Pena-Garay, JHEP 0204, 007 (2002) [hep-ph/0111150].

[9] A. N. Ioannisian and A. Y. Smirnov, Phys. Rev. Lett. 93, 241801 (2004); A. N. Ioannisian, N. A. Kazarian, A. Y. Smirnov and D. Wyler, Phys. Rev. D 71, 033006 (2005);

[10] A. N. Ioannisian and A. Y. Smirnov, Nucl. Phys. B 816, 94 (2009);

[11] A. N. Ioannisian and A. Y. Smirnov, arXiv:hep$\mathrm{ph} / 0201012$.

[12] E. Kh. Akmedov, M. A. Tortola, and J. W. F. Valle, JHEP 0405, 057 (2004), e-Print arXiv:hep-ph/0404083.

[13] S. S. Aleshin, O. G. Kharlanov and A. E. Lobanov, Phys. Rev. D 87, 045025 (2013) [arXiv:1302.7201 [hep-ph]].

[14] S. S. Aleshin, O. G. Kharlanov and A. E. Lobanov, arXiv:1110.5471 [hep-ph].

[15] Y. F. Li, J. Cao, Y. Wang and L. Zhan, Phys. Rev. D 88, 013008 (2013) [arXiv:1303.6733 [hep-ex]]; Y. F. Li, Int. J. Mod. Phys. Conf. Ser. 31, 1460300 (2014) [arXiv:1402.6143 [physics.ins-det]]. ?

[16] M. Wurm et al. [LENA Collaboration], arXiv:1104.5620 [astro-ph.IM].

[17] J. R. Alonso, N. Barros, M. Bergevin, A. Bernstein, L. Bignell, E. Blucher, F. Calaprice and J. M. Conrad et al., arXiv:1409.5864 [physics.ins-det].

[18] J. Kersten and A. Yu. Smirnov, to appear.

[19] P. M. Shearer, (2013) Upper Mantle Seismic Discontinuities, in Earth's Deep Interior: Mineral Physics and Tomography From the Atomic to the Global Scale (eds S.-I. Karato, A. Forte, R. Liebermann, G. Masters and L. Stixrude), American Geophysical Union, Washington, D. C.. doi: 10.1029/GM117p0115.

[20] N. Petersen, et al., Sharpness of the mantle discontinuities, Geophys. Res. Lett., 20, 859-862,1993.

[21] E. Lisi and D. Montanino, Phys. Rev. D 56, 1792 (1997) [hep-ph/9702343].

[22] S. P. Mikheyev and A. Y. Smirnov, Prog. Part. Nucl. Phys. 23 (1989) 41.

[23] L. Oberauer, Nucl. Phys. Proc. Suppl. 235-236, 198 (2013).

[24] M. Wurm, B. Caccianiga, D. D'Angelo, S. Davini, F. von Feilitzsch, M. Goger-Neff, T. Lachenmaier and T. Lewke et al., Phys. Rev. D 83, 032010 (2011) [arXiv:1012.3021 [astro-ph.IM]].

[25] R. Mollenberg, F. von Feilitzsch, D. Hellgartner, L. Oberauer, M. Tippmann, J. Winter, M. Wurm and V. Zimmer, Phys. Lett. B 737, 251 (2014) [arXiv:1408.0623 [physics.ins-det]].

[26] M. Wurm, B. Caccianiga, D. D’Angelo, S. Davini, F. von Feilitzsch, M. Goger-Neff, T. Lachenmaier and T. Lewke et al., Phys. Rev. D 83, 032010 (2011) [arXiv:1012.3021 [astro-ph.IM]].

[27] N. R. Lomb, Astrophys. Space Sci. 39, 447 (1976).

[28] J. D. Scargle, Astrophys. J. 263, 835 (1982).

[29] G. Ranucci and M. Rovere, Phys. Rev. D 75, 013010 (2007) [hep-ph/0605212].

[30] Indeed, the influence of matter on the 1-3 mixing is determined by $\epsilon\left(\Delta m_{31}^{2}\right) \sim 7 \cdot 10^{-5}$ and the observable effects are further suppressed by smallness of $s_{13}$ and averaging of oscillations associated with the third neutrino. For $\Delta m_{31}^{2}$ the oscillation length is smaller than $1 \mathrm{~km}$. Interference between the modes of oscillations inside the Earth driven by $\Delta m_{31}^{2}$ and $\Delta m_{21}^{2}$ produces a negligible effect. Thus we will use the vacuum value of $1-3$ mixing. 